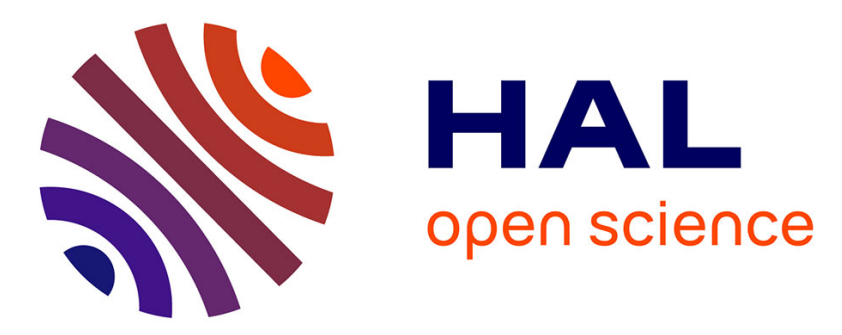

\title{
Second harmonic measurement of the order parameter variation near the multicritical point of $\mathrm{NH} 4 \mathrm{Cl}$
}

\author{
G. Dolino, J. P. Pique, M. Vallade
}

\section{To cite this version:}

G. Dolino, J. P. Pique, M. Vallade. Second harmonic measurement of the order parameter variation near the multicritical point of NH4Cl. Journal de Physique Lettres, 1979, 40 (13), pp.303-306. 10.1051/jphyslet:019790040013030300 . jpa-00231629

\section{HAL Id: jpa-00231629 https://hal.science/jpa-00231629}

Submitted on 1 Jan 1979

HAL is a multi-disciplinary open access archive for the deposit and dissemination of scientific research documents, whether they are published or not. The documents may come from teaching and research institutions in France or abroad, or from public or private research centers.
L'archive ouverte pluridisciplinaire HAL, est destinée au dépôt et à la diffusion de documents scientifiques de niveau recherche, publiés ou non, émanant des établissements d'enseignement et de recherche français ou étrangers, des laboratoires publics ou privés. 


\title{
Second harmonic measurement of the order parameter variation near the multicritical point of $\mathrm{NH}_{4} \mathrm{Cl}$
}

\author{
G. Dolino, J. P. Pique and M. Vallade \\ Université Scientifique et Médicale de Grenoble, \\ Laboratoire de spectrométrie physique (*), B.P. 53, 38041 Grenoble Cedex, France
}

(Reçu le 2 avril 1979, accepté le 8 mai 1979)

\begin{abstract}
Résumé. - On a mesuré les variations du paramètre d'ordre $\eta$ de $\mathrm{NH}_{4} \mathrm{Cl}$ en fonction de la pression par génération de la seconde harmonique de la lumière. La transition est continue au-dessus de 1,5 kbar. En-dessous, une coexistence de phase est observée qui induit des déformations plastiques. On discute les interprétations possibles des données à l'aide d'exposants critiques et de la théorie de Landau.
\end{abstract}

\begin{abstract}
Pressure variations of the order parameter $\eta$ of $\mathrm{NH}_{4} \mathrm{Cl}$ are measured by second harmonic generation of light. The transition is continuous above $1.5 \mathrm{kbar}$; below, a phase coexistence is observed which may induce plastic deformations. Possible interpretations of the data with critical exponents and with the Landau theory are discussed.
\end{abstract}

Multicritical points have been the subject of growing interest in the last few years and particularly since the work of Griffiths [1]. A tricritical point may be encountered when a transition changes from first order to second order as a function of an external parameter. Theoretical considerations [2] based on scaling hypotheses show that, for an Ising system, the tricritical behaviour is classical for $d \geqslant 3$ so that in many practical cases where $d=3$ one expects the mean field theory to be valid. However renormalization group theory predicts some logarithmic corrections and it has also been shown very recently that deviations from phenomenological theory are to be expected for critical amplitude ratios [3]. Various physical systems have been experimentally investigated to check these theories and in particular $\mathrm{He}_{3}-\mathrm{He}_{4}$ mixtures, metamagnets, ferroelectrics [4] and ammonium halides $[5,6,7]$. Most of the experiments are in agreement with a classical behaviour. However in ammonium chloride, which has a multicritical point at high pressure, the order parameter exponent $\beta$ measured at ambient pressure has been found to be smaller than its expected value near a tricritical point $[8,9,10]$. Some authors have interpreted these results as evidence for a higher order critical point [8-11]. This point is supported by recent heat capacity measurements which however do not clearly resolve the nature of the multicritical point [12-13]. Order parameter measure-

(*) (Associé au C.N.R.S.). ments under pressure have been made in $\mathrm{ND}_{4} \mathrm{Cl}$ by neutron-diffraction and small values of $\beta$ have also been reported [14].

In this letter we report the first investigation of the temperature and pressure variations of the order parameter in $\mathrm{NH}_{4} \mathrm{Cl}$. In this crystal the transition corresponds to a parallel ordering of the $\mathrm{NH}_{4}^{+}$tetrahedra and it is generally described as a compressible Ising model [15].

For our measurements we used second harmonic generation (S.H.G.) of light, a technique well suited for studying transformation from a centrosymmetric to a non centrosymmetric structure and which was first used by I. Freund in $\mathrm{NH}_{4} \mathrm{Cl}$ [16]. In this crystal a S.H. intensity $I \propto\left|d_{14} E_{x} E_{y}\right|^{2}$ is produced in the ordered phase, under the action of the light electric field $E$ of a YAG laser beam; $d_{14}$ is the nonlinear susceptibility coefficient which can be taken proportional to the order parameter $\eta$. The optical set-up was similar to that used in a study of the quartz transition [17]. The sample was a $4 \times 4 \times 6 \mathrm{~mm}^{3}$ single crystal grown from aqueous solution and cut with its large faces perpendicular to $\langle 110\rangle$ directions; it was placed in a four window high pressure cell with pentane as pressure fluid. The temperature cell was held constant within $10^{-2}{ }^{\circ} \mathrm{C}$ during a run and the pressure, measured with a sensitivity of 0.2 bar, was changed step by step.

Simultaneously with the S.H.G. measurements the small angle linear scattering of the laser beam was 


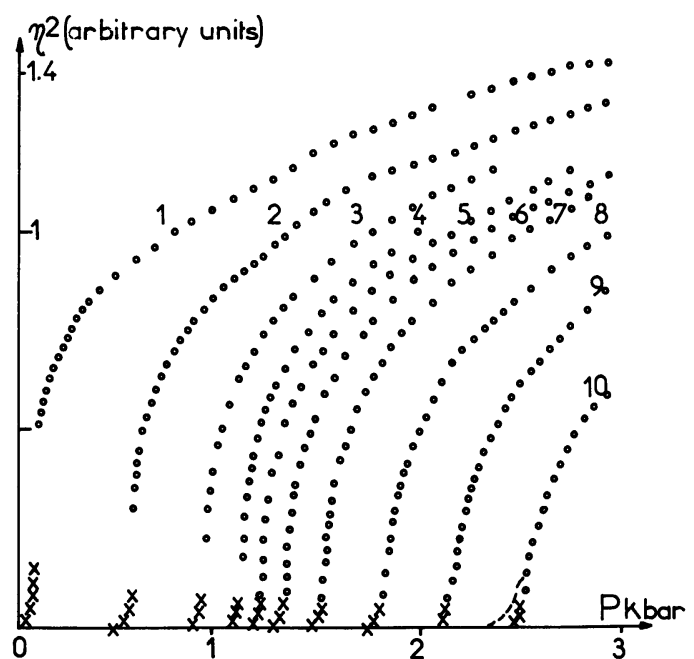

Fig. 1. - Pressure dependence of the second harmonic intensity (proportional to the square of the order parameter) for the 10 different temperatures given in table I. The experimental points plotted as crosses correspond to the residual order and have not been taken into account in the fitting procedures. The dashed line on the curve 10 shows the after-effect due to irreversible damage induced by the 1st order transition.

measured and the sample was observed with a microscope. The experimental curves of S.H. intensity versus pressure for 10 different temperatures are plotted in figure 1. Two main questions arise in interpreting these intensity variations :

a) Proportionality OF S.H. INTENSITY to $\eta^{2}(P, T)$ IN THE ORDERED PHASE. - Although $d_{14}$ is expected to be proportional to $\eta$, the S.H.G. is greatly influenced by the domain structure [18] and in particular harmonic diffraction is observed [16]. Nevertheless, the intensity integrated over the small angle diffracted peaks appear to be weakly dependent on the detailed domain structure in the present case, probably because the sample possesses a large number of domains of randomly distributed sizes.

The shape of the curves for a constant $T$ is quite reproducible from run to run (to within $2 \%$ ) although the absolute intensity may vary by about $20 \%$. (The curves in figure 1 have been normalized by performing a run at a constant pressure of 2800 bars.) The proportionality between S.H. intensity and $\eta^{2}(P, T)$ is also supported by a comparison of the various measurements of the order parameter variations at 1 bar performed on single domain or polydomain samples [10].

b) BEHAVIOUR NEAR THE TRANSITION POINT. - As in most of the previous experiments $[8,9,10]$, one observes a tail of residual order in the disordered phase. Microscope observations show numerous slide bands perpendicular to $\langle 100\rangle$ type directions which may well be responsible of the smearing of the transition [18]. Corresponding to these defects, small angle scattering with a maximum intensity at the transition point is observed and we think that the light scattering observed by Fritz et al. [7] may well have the same origin. Above $1.5 \mathrm{kbar}$ no hysteresis is found and the transition is continuous. Below this pressure hysteresis appears and a mixing of the ordered and disordered phases is observed as nuclei elongated perpendicularly to $\langle 111\rangle$ type directions [18]. These nuclei give rise to additional small angle scattering near $T_{\mathrm{c}}$, which is rather weak between $1.5 \mathrm{kbar}$ and $1 \mathrm{kbar}$ but becomes so strong for lower pressures that the transmitted beam may be attenuated by $70 \%$.

This increase in scattering is interpreted as arising from large plastic deformations produced by the coexistence of two phases with different lattice parameters [19]. Irreversible damage are thus produced when passing the discontinuous transition at low pressure and anomalies are then observed near $T_{\mathrm{c}}$ after such a treatment. (See the bump on the dotted curve in figure 1.) Therefore most of the data in figure 1 have been obtained on a virgin sample which has gone in the ordered phase only through a continuous transition.

In order to interpret the experimental curves $\eta(P, T)$, we have tried least square fitting procedures with different theoretical formulas.

We first attempted a power law of the form

$$
\eta=K\left(P-P^{*}\right)^{\beta}
$$

for each temperature. The results obtained are given in table I. The effective exponent $\beta$ increases from 0.124 to 0.270 with the mean square deviation $\sigma$ increasing from 0.003 to 0.01 in the 2 nd order region. Crossover effects [20] may explain this behaviour but the data are not sufficiently accurate, in particular near the transition, to justify the introduction of additional fitting parameters.

For temperatures near the multicritical point we have tried a fit with :

$$
\eta=A\left(P-P_{\mathrm{c}}\right)^{1 / 4}\left[\operatorname{Ln}\left(\frac{P-P_{\mathrm{c}}}{B}\right)\right]^{1 / 4} \text {. }
$$

This corresponds to the formula obtained from renormalization group theory for a tricritical point $[2,21]$. A good agreement $(\sigma=0.0024)$ is indeed obtained for the curve at

$$
T_{\mathrm{c}}=-16.95^{\circ} \mathrm{C} \text { with } B=10.200 \text { bars } .
$$

As previously noted by Yelon et al. [14] for $\mathrm{ND}_{4} \mathrm{Cl}$, the introduction of a logarithmic correction gives an effective $\beta \sim 0.182$.

As an alternative to these power laws we have tried fits with a Landau mean field theory [22]. The usual free energy is written, for a given temperature $T$ :

$$
\begin{aligned}
F=-\left(P-P_{0}(T)\right) \frac{\eta^{2}}{2}+ & \frac{b(T)}{4} \eta^{4}+ \\
& +\frac{c}{6} \eta^{6}+\frac{d}{8} \eta^{8}+\cdots
\end{aligned}
$$


Table I. - Results of fits to the order parameter variations for the 10 different isotherms investigated.

A. Power law $\eta \sim\left(P-P^{*}\right)^{\beta}$.

B. Landau theory, eq. (2) (with $c=0$ and $d=955.5$ ).

C. Landau theory, eq. (3) (with $b^{\prime}=0.621$ and $c=405$ ).

The pressures are in bars and the coefficient $b, c, d$ correspond to an arbitrary normalization of $\eta$. There is a $67 \%$ probability that the parameters have a value inside the given uncertainty (for the data used in these fits). The transition, with decreasing pressure occurs from 0 to 2 bars below the lower limit of the indicated pressure range, except for run (1) and (9) for which the transition pressures are 110 and 2134 bars respectively.

\begin{tabular}{|c|c|c|c|c|c|c|c|c|c|c|c|}
\hline & & & A & & & & & & & ( & \\
\hline$T^{\circ} \mathrm{C}$ & Pressure range & & & $\beta\left(\times 10^{3}\right)$ & $P_{0}$ & & $b$ & & & $P_{0}$ & $P_{0}-P_{1}$ \\
\hline & - & & & - & & & & & & & - \\
\hline-29.47 & $2927-$ & 65 & \pm 4 & $124 \pm 2$ & 115 & \pm 18 & -272 & \pm 30 & 90 & \pm 10 & $382 \pm 35$ \\
\hline-25.04 & $2927-$ & 576.5 & \pm 0.9 & $131 \pm 1$ & 625 & \pm 4 & -219 & \pm 12 & 587 & \pm 3 & $215 \pm 15$ \\
\hline-21.87 & $2573-$ & 936.0 & \pm 0.2 & $143 \pm$ & 957 & 1 & -120 & \pm 5 & 943.5 & 0.9 & $172 \pm 6$ \\
\hline-20.29 & $1854-1123.1$ & 1120.4 & \pm 0 & 149 & $1132.4 \pm$ & 0.9 & -82 & \pm & 1127.8 & 0.7 & $164 \pm$ \\
\hline-19.47 & $2534-1221.7$ & 1220.92 & \pm 0.08 & & $1222.7 \pm$ & 0.7 & 19.4 & 2.4 & 1224.8 & 0.2 & $113 \pm$ \\
\hline-18.47 & $2927-1335.9$ & & & & & 0.4 & 19.6 & 3.2 & 6.05 & 0.16 & $62 \pm$ \\
\hline-16.95 & $2883-1517.4$ & 1516.8 & & & $1514.0 \pm$ & 0.3 & 40.1 & 2.2 & 1516.8 & 0.1 & $35 \pm$ \\
\hline-14.45 & $2928-1814.0$ & 1812.4 & & $210=$ & $1803.8 \pm$ & 0.3 & 124 & 2 & 1808.4 & 0.3 & $64 \pm$ \\
\hline-11.83 & $2927-2149.7$ & 2143.9 & \pm & $225 \pm 4$ & $2123.2 \pm$ & 0.7 & 236 & \pm 4 & 2133.0 & 0.5 & $-178 \pm$ \\
\hline-8.99 & $2927-2505.9$ & 2503.8 & \pm 0.5 & $272 \pm 4$ & $2490.6 \pm$ & \pm 1 & 292 & \pm 10 & 2497.5 & \pm 0.4 & $-269 \pm$ \\
\hline
\end{tabular}

Restricting to 3 the number of coefficients in this expansion, we obtain bad fits for an ordinary tricritical point $(c \neq 0$ and $d=0)$, good ones with $c=0$ and $d \neq 0$ (fits with $d \neq 0, b=0$ and $c$ function of $T$ are a little less good). These fits with $d \neq 0$ correspond to a higher order critical point of order 4 [9-11]. (The introduction of the $\eta^{10}$ term with $c=d=0$ works well only for our 3 lowest temperatures.) One may note that equation (2) implies a somewhat arbitrary dissymmetry in the role of the pressure and the temperature. As the coefficients of $\eta^{2}$ and of $\eta^{4}$ must vanish at the multicritical point, they can both be linearly expanded as a function of $P$ and $T$ near this point [23]. For a constant temperature this leads to a pressure dependent $b=b^{\prime}\left(P-P_{1}(T)\right)$ and making $d=0$ in (2), one gets :

$$
\begin{aligned}
& \eta^{2}(P, T)=\frac{-b^{\prime}}{2 c}\left[\left(P-P_{1}(T)\right)-\right. \\
& \left.\quad-\left(\left(P-P_{1}(T)\right)^{2}+\frac{4 c}{b^{\prime 2}}\left(P-P_{0}(T)\right)\right)^{1 / 2}\right] .
\end{aligned}
$$

A somewhat unexpected feature of this formula is that it presents a saturation of $\eta$ for large $P$ and it thus provides a better description of the order parameter on a larger range of pressure than in the usual Landau expansion (eq. (2) with $d=0$ ). Fits will all the parameters $P_{0}, P_{1}, b^{\prime}$ and $c$ free, give small $\sigma(0.003$ near $T_{\mathrm{t}}$ with only slight variation of $b^{\prime}$ and $c$ so that these coefficients can be taken to be constant (table I)).

The variations of $P_{0}$ and $P_{1}$ with $T$ so obtained, are nearly linear, which is consistent with the above assumption of a linear expansion of the coefficients of $\eta^{2}$ and $\eta^{4}$ as a function of temperature and pressure. Eq. (3) was also found to describe quite well recent data near the tricritical point in $\mathrm{KH}_{2} \mathrm{PO}_{4}$ [24].

In conclusion our analysis shows clearly the difficulty in making a definite choice between various kinds of description of the critical behaviour in $\mathrm{NH}_{4} \mathrm{Cl}$. The points which have been firmly established can be summarized as follows :

- The multicritical point is found near $1.5 \mathrm{kbar}$; in the first order region plastic deformations appear when the volume discontinuity is too large, which influence the ordering near $T_{\mathrm{c}}$.

- The description of the pressure and temperature dependence of the order parameter, with a single effective exponent is not very satisfactory in the whole range studied.

- A consistent description of all the curves is obtained with Landau free energy expansions either with a $\eta^{8}$ term and the $\eta^{4}$ coefficient dependent only on $T$ or with a $\eta^{6}$ term and the $\eta^{4}$ coefficient depending linearly on $T$ and $P$. The former case corresponds to a critical point of order 4 ; the latter to a tricritical one. Although one cannot really choose between the two hypotheses only on the basis of our present data, the tricritical point description is more attractive since it does not require a somewhat arbitrary vanishing of one coefficient in expansion (2) and it does not introduce a dissymmetry between $P$ and $T$. Such a dissymmetry appears to be rather artificial since in the theory of scaling laws the true scaling fields are combinations of these two parameters [25]. 


\section{References}

[1] Griffiths, R. B., Phys. Rev. Lett. 24 (1970) 715.

[2] Wegner, F. J. and Riedel, E. K., Phys. Rev. B 7 (1973) 248

[3] Fisher, M. E. and Sarbach, S., Phys. Rev. Lett. 41 (1978) 1127.

[4] Bastie, P., Vallade, M., Vettier, C. and Zeyen, C. M. E., Phys. Rev. Lett. 40 (1978) 337 and references therein.

[5] Garland, C. W. and Weiner, B. B., Phys. Rev. B 3 (1971) 1634 Weiner, B. B. and Garland, C. W., J. Chem. Phys. 56 (1972) 155.

[6] Mandema, W. and Trappeniers, N. J., Physica 76 (1974) 123.

[7] Fritz, J. and Cummins, H. Z., Phys. Rev. Lett. 28 (1972) 96.

[8] Bruins, D. E. and Garland, C. W., J. Chem. Phys. 63 (1975) 4139.

[9] Steinbrener, S. and Jahn, I. R., J. Phys. C. 11 (1978) 1337. SteInbrener, S., Solid State Commun. 26 (1978) 957.

[10] Pique, J. P., Dolino, G. and Vallade, M., J. Physique 39 (1978) 1003.

[11] Benguigui, L., Solid State Commun. 20 (1976) 173.

[12] Garland, C. W. and Baloga, J. D., Phys. Rev. B 16 (1977) 331

[13] Amitin, E. B., Kobalevskaya, Y. A. and Paukov, I. E., $Z h$. Eskp. Teor. Fiz. 71 (1976) 700 (Sov. Phys. JETP 44 (1976) 368).

[14] Yelon, W. B., Cox, D. E., Kortman, P. J. and Daniels, W. B., Phys. Rev. B 9 (1974) 4843.

[15] For discussion of the mechanism of the transition on $\mathrm{NH}_{4} \mathrm{Cl}$ see :
Huller, A., Z. Phys. 254 (1972) 456

Huller, A., Z. Phys. 270 (1974) 343 ;

Vaks, V. G. and Shneider, V. E., Phys. Status Solidi A 35 (1976) 61.

[16] Freund, I., Phys. Rev. Lett. 19 (1967) 1288; Phys. Rev. Lett. 21 (1968) 1404.

Freund, I. and Kopf, L., Phys. Rev. Lett. 24 (1970) 1017.

[17] Bachheimer, J. P. and Dolino, G., Phys. Rev. B 11 (1975)

[18] Pique, J. P., Dolino, G. and Vallade, M., J. Physique 38 (1977) 1527.

[19] This plastic deformation may be the origin of the self polarization observed by TAYlor, B. E., Miller, M. G. and LASKar, A. L., Phys. Rev. Lett. 40 (1978) 1101.

[20] Riedel, E. K. and Wegner, F. J., Phys. Rev. B 9 (1974) 294.

[21] Aharony, A. and Halperin, B. I., Phys. Rev. Lett. 35 (1975) 1308.

[22] LANDAU, L. and LIFCHITZ, E., Physique statistique (MIR, Moscou) 1967.

[23] Poppe, U. and Huller, A., J. Phys. C. 11 (1978) 245.

[24] Bastie, P., Vallade, M., Vettier, C., Zeyen, C. M. E. and MEISTER, H. (Unpublished results).

[25] Griffiths, R. B. and Wheeler, J. C., Phys. Rev. A 2 (1970) 1047. 